\title{
Statin Gene Testing: Revolutionary Tool to Increase Compliance and Care in Patients with Statin-Induced Myopathy
}

\author{
Jacob C Vinson ${ }^{1}$, Parivash Badar ${ }^{2 *}$, Deepika Gopalakrishnan ${ }^{2}$, Ambarish Gopal ${ }^{2}$ \\ ${ }^{1}$.Baylor Scott \& White - The Heart Group, Plano TX \\ ${ }^{2}$ The Heart Hospital Baylor Plano. Plano TX
}

\begin{abstract}
Modern day practitioners are utilizing their time and efforts increasingly to focus on preventative medicine. The correlation between coronary artery disease and hyperlipidemia is well established and this correlation has helped mold the field of preventative cardiovascular medicine. Clinically, patients treated with statins as per the guidelines have shown lower rates of morbidity and mortality over multiple large scale trials [2,4]. Statins have thus become a major first line therapy for treating patients with known hyperlipidemia and coronary artery disease; however, statin medications are often discontinued in a clinical setting with nearly $75 \%$ of patients discontinuing therapy within 2 years of initiation [5,7]. The 2009 SEARCH trial first identified an association between the pharmacogenetic test for solute carrier organic anion transporter family member 1B1 (SLCO1B1) and statin-associated symptoms, and has been replicated among numerous other studies, particularly with simvastatin [9-12]. There is some evidence that suggests the implication of SLCO1B1 results can change the prescribing pattern for providers [15]. With the use of this testing technology, clinicians are able to identify and predict possible statin-induced myopathy side effects in patients, increase compliance, and provide a more personalized treatment regimen.
\end{abstract}

Keywords: Statin, Cardiovascular disease, Vitamin D, SLCO1B1, Statin-associated myopathy, coronary artery disease, management, prevention, hyperlipidemia.

\section{Introduction}

Modern day practitioners are utilizing their time and efforts increasingly to focus on preventative medicine. The correlation between coronary artery disease and hyperlipidemia is well established and this correlation has helped mold the field of preventative cardiovascular medicine. Clinically, patients treated with statins as per the guidelines have shown lower rates of morbidity and mortality over multiple large scale trials [2,4]. Statins have thus become a major first line therapy for treating patients with known hyperlipidemia and coronary artery disease. Many trials and observational studies have put forward information regarding muscle injury and statin use over the years. About $60 \%$ of patients who discontinued statins, cited muscular pain as the main reason for discontinuation. These injuries range from mild myalgias to clinically significant myonecrosis and rhabdomyolysis. However, these side effects have led to low compliance rates and insufficient effect [17].

\section{Discussion:}

Physicians working towards prevention of cardiovascular disease start by monitoring the pertinent risk factors, such as smoking, lifestyle, cholesterol levels, hypertension, diabetes, obesity, etc. Total cholesterol (TC) and low-density lipoprotein cholesterol (LDL) are among the most important risk factors for cardiovascular disease when found at elevated levels in blood plasma, with elevated LDL leading to a buildup of plaques lining the lumen of arteries. Therefore, high LDL concentrations are linked with an increased risk for cardiovascular disease, such as coronary artery disease and stroke [1,2].

Corresponding Author: Parivash Badar, The Heart Hospital Baylor Plano. Plano TX. Received date: June 14, 2019; Accepted date: June 21, 2019; Published date: June 24, 2019.

DOI: https://doi.org/10.31546/JCCCVT.1004 
Over 100 million adults have elevated LDL levels in the United States, with less than half of those patients receiving any type of treatment. Among the patients that do receive some type of treatment, less than $35 \%$ achieve adequate control [2]. Statins are the first choice of pharmacological treatment to reduce LDL among physicians by reducing TC and LDL via inhibition of HMG-CoA reductase in the cholesterol biosynthesis pathway [3,4]. This reduction is associated with lowering the risk of developing cardiovascular disease in patients with high levels of LDL, as well as the mortality and disease progression for patients with atherosclerotic cardiovascular disease (ASCVD) [4].

The ACC/AHA guidelines recommends statin therapy for 4 classes of patients: 1. Patients with vascular diseases, 2. Patients with LDL $>190 \mathrm{mg} / \mathrm{dl}$, 3. Patients, age 40-75 with Diabetes Mellitus (DM), with LDL 70-189 mg/dl, 4. Patients, age 40-75 with LDL 70-189 $\mathrm{mg} / \mathrm{dl}$, and a calculated ASCVD risk score dependent on hypertension, smoking history, family history, obesity, etc. Primary prevention for statins would be for patients with an estimated 10 year risk of ASCVD >7.5\% and secondary prevention for patients with known ASCVD, LDL $>190 \mathrm{mg} / \mathrm{dl}$ or DM/Chronic Kidney Disease [3,13].

These guidelines recommend starting with highintensity statin therapy, which lowers LDL by $\geq 50 \%$ via Atorvastatin 40 or $80 \mathrm{mg}$ and Rosuvastatin 20 or $40 \mathrm{mg}$. Patients $>75$ years, with statin intolerance, liver disease or kidney disease are treated with moderate-intensity statin therapy, which lowers LDL by $30-49 \%$ via Atorvastatin 10 or $20 \mathrm{mg}$, Rosuvastatin 5 or $10 \mathrm{mg}$, Simvastatin 20 or $40 \mathrm{mg}$, Pravastatin 40 or $80 \mathrm{mg}$, Lovastatin $40 \mathrm{mg}$, and Fluvastatin $40 \mathrm{mg}$ BID. Both therapies require assessment of dose response and tolerance within 6-8 weeks. If LDL is adequately reduced, then regular interval monitoring of risk factors and compliance are necessary [13,16]. Lipids are assessed on an annual basis, Hemoglobin A1c every 3 months for patients with DM, and Liver Function Testing/Creatine Kinase are checked in patients with previous history of liver disease or elevated liver enzymes.

Statin medications are often discontinued in a clinical setting with nearly $75 \%$ of patients discontinuing therapy within 2 years of initiation. Among these patients, $65 \%$ stated the main reason for non-adherence or discontinuation was side effects, with musculoskeletal symptoms dominating at roughly $40 \%[5,7,17]$. Other side effects include GI discomfort, fatigue, liver enzyme elevation and, in more rare occurrences, myositis - muscle symptoms with elevated serum creatine kinase (CK) levels [7]. Physicians are therefore discontinuing statin medications on a routine basis, but with a majority of clinicians rechallenging with low-intensity statin therapy or using at least two statins before determining that a patient has statin-associated symptoms [6].
This is consistent with current recommendations as the European Atherosclerosis Society (EAS) consensus panel recommends rechallenging with at least three statins attempted, while the National Lipid Association (NLA) recommends at least two statins, to identify patients with true statin-associated symptoms $[7,8]$.

The 2009 SEARCH trial first identified an association between the pharmacogenetic test for solute carrier organic anion transporter family member 1B1 (SLCO1B1) and statin-associated symptoms, and has been replicated among numerous other studies, particularly with simvastatin [9-12]. About 25\% of people carry either one or two copies of the SLCO1B1 variant This increases their risk up to 4.5 -fold - or 17fold respectively for developing significant statin induced myopathy $[9,17]$. Few reports, however, report patient outcomes after the delivery of these pharmacogenetic results, with the most notable finding being a pilot trial that observed short-term improvements in LDL after SLCO1B1 testing (10 $\mathrm{mg} / \mathrm{dl}$ decrease in LDL) [14]. However, there is some evidence that suggests the implication of SLCO1B1 results can change the prescribing pattern for providers (15).

Based on the SLCO1B1 testing results and genotype determination, therapy is initiated as following:

1. T/T genotype: Standard doses of statins, if indicated, are recommended

2. T/C genotype: Moderate to low doses of water soluble statins (in order of solubility: pravastatin, pitavastatin, rosuvastatin or fluvastatin), if indicated, are recommended

3. C/C genotype: Low doses of water soluble statins (in order of solubility: pravastatin, pitavastatin, rosuvastatin or fluvastatin), if indicated, are recommended [9].

This personalized testing method allows patients to uncover their genetic make-up and the factors influencing it. These genetic tests not only focus on the statin gene but other genetic factors influencing it. An inverse relationship has been established between the levels of Vitamin D and severity of coronary artery disease by multiple large scale research trials [18]. Along with that, an association of low levels of vitamin $\mathrm{D}$ and statin induced myopathy has also been established. Patients with low levels of Vitamin D are predisposed to higher levels of myopathy and may even undergo rhabdomyolysis more readily when compared to patients with normal Vitamin D levels. Vitamin D, thus works at gene level and regulates the mechanism of other factors influencing myopathy in patients on statin. Physicians treating patients with these symptoms must have this aspect in mind and should routinely check their patients Vitamin D levels to ensure avoidance of such symptoms. 
Historically, there have been several companies which provide a platform for patients to have a comprehensive blood panel with SLCO1B1 and Vitamin D level testing if requested by the provider. This genetic association has helped changing providers prescribing pattern; moreover, the results of this testing may provide further opportunity to reduce the amount of non-compliance by the patients. This effect can lead to a revolutionary change in the management and prevention of cardiovascular diseases.

\section{Conclusion:}

The use of statin gene, SLCO1B1 and Vitamin D levels can improve the level of care received at a cardiovascular clinic and decrease morbidity and mortality associated with coronary artery disease. With the use of this technology, clinicians are able to identify and predict possible statin induced myopathy side effects in patients who may have the particular genotype. This can be helpful to increase compliance in such patients and provide a more personalized treatment regimen. We hope to see more studies in the future that will further shed light on this topic.

\section{Conflict of Interest: None.}

\section{References}

1. Catapano AL, et al.Corrigendum to: 2016 ESC/EAS Guidelines for the Management of Dyslipidaemias. Eur Heart J. 2017; 39.1254.

2. Kuklina, EV, et al. Vital Signs: Prevalence, Treatment, and Control of High Levels of Low-Density Lipoprotein Cholesterol --- United States, 1999-2002 and 20052008. MMWR. 2011; 60:109-114.

3. Stone NJ, et al. 2013 ACC/AHA Guideline on the Treatment of Blood Cholesterol to Reduce Atherosclerotic Cardiovascular Risk in Adults. Circulation. 2014;129:S1-S45.

4. Taylor F, et al. Statins for the Primary Prevention of Cardiovascular Disease (Update). Cochrane Database Syst Rev. 2013;1:CD004816.

5. Zhang Huabing, et al. Discontinuation of Statins in Routine Care Settings. Ann Intern Med. 2013; 158: 526534.

6. Hovingh GK, et al. Identification and Management of Patients with Statin-Associated Symptoms in Clinical Practice: A Clinician Survey. Atherosclerosis. 2016; 245:111-117.
7. Stroes ES, et al. Statin-Associated Muscle Symptoms: Impact on Statin Therapy-European Atherosclerosis Society Consensus Panel Statement on Assessment, Aetiology and Management. Eur Heart J. 2015; 36:1012-1022.

8. Bays H, et al. An Assessment by the Statin Liver Safety Task Force: 2014 Update. J Clin Lipidol. 2014; 8:S47-S57.

9. Link E, et al. SLCO1B1 variants and statin-induced myopathy--a genomewide study N Engl J Med. 2008; 359:789-99.

10. Donnelly LA, et al. Common Nonsynonymous Substitutions in SLCO1B1 Predispose to Statin Intolerance in Routinely Treated Individuals With Type 2 Diabetes: A Go-DARTS Study. Clin Pharmacol Ther. 2011; 89:210-216.

11. Puccetti L, Ciani F, Auteri A. Genetic Involvement in Statins Induced Myopathy. Preliminary Data from an Observational Case-Control Study. Atherosclerosis. 2010; 211:28-29.

12. Ramsey LB, et al. The Clinical Pharmacogenetics Implementation Consortium Guideline for SLCO1B1 and Simvastatin-Induced Myopathy: 2014 Update. Clin Pharmacol Ther. 2014;96:423-8.

13. Rubenfire, Melvyn. 2019 ACC/AHA Guideline on the Primary Prevention of Cardiovascular Disease. J Am Coll Cardiol. 2019.

14. Baigent C, et al. Efficacy and Safety of Cholesterol-Lowering Treatment: Prospective Meta-Analysis of Data from 90,056 Participants in 14 Randomised Trials of Statins. Lancet. 2005; 366:1267-1278.

15. Vassy JL, et al. Impact of SLCO1B1 Pharmacogenetic Testing on Patient and Healthcare Outcomes: A Systematic Review. Clin. Pharmacol. Ther. 2018 Aug 23.

16. Adolphe A. Lipid Guidelines 2018: Updates from ACC/AHA Guidelines 2013. American College of Physicians. 2018.

17. Wei MY, et al. Predictors of Statin Adherence, Switching, and Discontinuation in the USAGE Survey: Understanding the Use of Statins in America and Gaps in Patient Education. J Clin Lipidol. 2013; 7:472-83.

18. Sogomonian R, et al. Serum Vitamin D Levels Correlate to Coronary Artery Disease Severity: a Retrospective Chart Analysis. Expert Rev Cardiovasc Ther. 2016; 14:977-982. 